\title{
A Case for Hierarchical Rings with Deflection Routing: An Energy-Efficient On-Chip Communication Substrate
}

\author{
Rachata Ausavarungnirun Chris Fallin Xiangyao Yu† Kevin Kai-Wei Chang \\ Greg Nazario Reetuparna Das§ Gabriel H. Loh $\ddagger$ Onur Mutlu \\ Carnegie Mellon University §University of Michigan †MIT †AMD \\ Corresponding author email's: rachata@cmu.edu Phone: 412-225-5919
}

\begin{abstract}
Hierarchical ring networks, which hierarchically connect multiple levels of rings, have been proposed in the past to improve the scalability of ring interconnects, but past hierarchical ring designs sacrifice some of the key benefits of rings by reintroducing more complex in-ring buffering and buffered flow control. Our goal in this paper is to design a new hierarchical ring interconnect that can maintain most of the simplicity of traditional ring designs (i.e., no in-ring buffering or buffered flow control) while achieving high scalability as more complex buffered hierarchical ring designs.

To this end, we revisit the concept of a hierarchical-ring network-on-chip. Our design, called HiRD (Hierarchical Rings with Deflection), includes critical features that enable us to mostly maintain the simplicity of traditional simple ring topologies while providing higher energy efficiency and scalability. First, HiRD does not have any buffering or buffered flow control within individual rings, and requires only a small amount of buffering between the ring hierarchy levels. When inter-ring buffers are full, our design simply deflects flits so that they circle the ring and try again, which eliminates the need for in-ring buffering. Second, we introduce two simple mechanisms that together provide an end-to-end delivery guarantee within the entire network (despite any deflections that occur) without impacting the critical path or latency of the vast majority of network traffic.

Our experimental evaluations on a wide variety of multiprogrammed and multithreaded workloads and synthetic traffic patterns show that HiRD attains equal or better performance at better energy efficiency than multiple versions of both a previous hierarchical ring design and a traditional single ring design. We also extensively analyze our design's characteristics and injection and delivery guarantees. We conclude that HiRD
\end{abstract}

Preprint submitted to Elsevier

November 5, 2015 
can be a compelling design point that allows higher energy efficiency and scalability while retaining the simplicity and appeal of conventional ring-based designs.

\section{Introduction}

Interconnect scalability, performance, and energy efficiency are first-order concerns in the design of future CMPs (chip multiprocessors). As CMPs are built with greater numbers of cores, centralized interconnects (such as crossbars or shared buses) are no longer scalable. The Network-on-Chip (NoC) is the most commonly-proposed solution [12]: cores exchange packets over a network consisting of network switches and links arranged in some topology.

Mainstream commercial CMPs today most commonly use ring-based interconnects. Rings are a well-known network topology [11], and the idea behind a ring topology is very simple: all routers (also called "ring stops") are connected by a loop that carries network traffic. At each router, new traffic can be injected into the ring, and traffic in the ring can be removed from the ring when it reaches its destination. When traffic is traveling on the ring, it continues uninterrupted until it reaches its destination. A ring router thus needs no in-ring buffering or flow control because it prioritizes onring traffic. In addition, the router's datapath is very simple compared to a mesh router, because the router has fewer inputs and requires no large, power-inefficient crossbars; typically it consists only of several MUXes to allow traffic to enter and leave, and one pipeline register. Its latency is typically only one cycle, because no routing decisions or output port allocations are necessary (other than removing traffic from the ring when it arrives). Because of these advantages, several prototype and commercial multicore processors have utilized ring interconnects: the Intel Larrabee [55], IBM Cell [51], and more recently, the Intel Sandy Bridge [27].

Unfortunately, rings suffer from a fundamental scaling problem because a ring's bisection bandwidth does not scale with the number of nodes in the network. Building more rings, or a wider ring, serves as a stopgap measure but increases the cost of every router on the ring in proportion to the bandwidth increase. As commercial CMPs continue to increase core counts, a new network design will be needed that balances the 


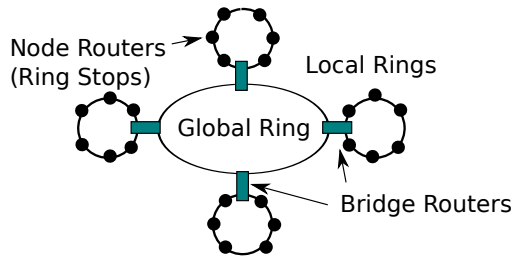

Figure 1: A traditional hierarchical ring design [53, 64, 24, 54, 22] allows "local rings" with simple node routers to scale by connecting to a "global ring" via bridge routers.

simplicity and low overhead of rings with the scalability of more complex topologies.

A hybrid design is possible: rings can be constructed in a hierarchy such that groups of nodes share a simple ring interconnect, and these "local" rings are joined by one or more "global" rings. Figure 1 shows an example of such a hierarchical ring design. Past works [53, 64, 24, 54, 22] proposed hierarchical rings as a scalable network. These proposals join rings with bridge routers, which reside on multiple rings and transfer traffic between rings. This design was shown to yield good performance and scalability [53]. The state-of-the-art design [53] requires flow control and buffering at every node router (ring stop), because a ring transfer can make one ring back up and stall when another ring is congested. While this previously proposed hierarchical ring is much more scalable than a single ring [53], the reintroduction of in-ring buffering and flow control nullifies one of the primary advantages of using ring networks in the first place (i.e., the lack of buffering and buffered flow control within each ring).

Our goal in this work is to design a ring-based topology that is simpler and more efficient than prior ring-based topologies. To this end, our design uses simple ring networks that do not introduce any in-ring buffering or flow control. Like past proposals, we utilize a hierarchy-of-rings topology to achieve higher scalability. However, beyond the topological similarities, our design is very different in how traffic is handled within individual rings and between different levels of rings. We introduce a new bridge router microarchitecture that facilitates the transfer of packets from one ring to another. It is in these, and only these, limited number of bridge routers where we require any buffering.

Our key idea is to allow a bridge router with a full buffer to deflect packets. Rather than requiring buffering and flow control in the ring, packets simply cycle through the 
network and try again. While deflection-based, bufferless networks have been proposed and evaluated in the past [5, 26, 56, 2, 46, 20], our approach is effectively an elegant hybridization of bufferless (rings) and buffered (bridge routers) styles. To prevent packets from potentially deflecting around a ring arbitrarily many times (i.e., to prevent livelock), we introduce two new mechanisms, the injection guarantee and the transfer guarantee, that ensure packet delivery even for adversarial/pathological conditions (as we discuss in [3] and evaluate with worst-case traffic in $\$ 4.3$.

This simple hierarchical ring design, which we call HiRD (for Hierarchical Rings with Deflection), provides a more scalable network architecture while retaining the key simplicities of ring networks (no buffering or flow control within each ring). We show in our evaluations that HiRD provides better performance, lower power, and better energy efficiency with respect to the buffered hierarchical ring design [53].

In summary, our major contributions are:

- We propose a new, low-cost, hierarchical ring NoC design based on very simple router microarchitectures that achieve single-cycle latencies. This design, HiRD, places an ordinary ring router (without flow control or buffering) at every network node, connects local rings with global rings using bridge routers, which have minimal buffering and use deflection rather than buffered flow control for inter-ring transfers.

- We provide new mechanisms for guaranteed delivery of traffic ensuring that inter-ring transfers do not cause livelock or deadlock, even in the worst case.

- We qualitatively and quantitatively compare HiRD to several state-of-the-art NoC designs. We show competitive performance to these baselines, with better energy efficiency than all prior designs, including, most importantly, the hierarchical ring design with in-ring buffering and buffered flow control [53]. We conclude that HiRD represents a compelling design point for future many-core interconnects by achieving higher performance while maintaining most of the simplicity of traditional ring-based designs. 


\section{HiRD: Simple Hierarchical Rings with Deflection}

In this section, we describe the operation of our network design HiRD, or Hierarchical Rings with Deflection. HiRD is built on several basic operation principles:

1. Every node (e.g., CPU, cache slice, or memory controller) resides on one local ring, and connects to one node router on that ring.

2. Node routers operate exactly like routers (ring stops) in a single-ring interconnect: locally-destined flits are removed from the ring, other flits are passed through, and new flits can inject whenever there is a free slot (no flit present in a given cycle). There is no buffering or flow control within any local ring; flits are buffered only in ring pipeline registers. Node routers have a single-cycle latency.

3. Local rings are connected to one or more levels of global rings to form a tree hierarchy.

4. Rings are joined via bridge routers. A bridge router has a node-router-like interface on each of the two rings it connects, and has a set of transfer FIFOs (one in each direction) between the rings.

5. Bridge routers consume flits that require a transfer whenever the respective transfer FIFO has available space. The head flit in a transfer FIFO can inject into its new ring whenever there is a free slot (exactly as with new flit injections). When a flit requires a transfer but the respective transfer FIFO is full, the flit remains in its current ring. It will circle the ring and try again next time it encounters the correct bridge router (this is a deflection).

By using deflections rather than buffering and blocking flow control to manage ring transfers, HiRD retains node router simplicity, unlike past hierarchical ring network designs. This change comes at the cost of potential livelock (if flits are forced to deflect forever). We introduce two mechanisms to provide a deterministic guarantee of livelock-free operation in [3].

While deflection-based bufferless routing has been previously proposed and evaluated for a variety of off-chip and on-chip interconnection networks (e.g., [5, 46, 20, 
[1, 19, 49, 50]), deflections are trivially implementable in a ring: if deflection occurs, the flit 1 continues circulating in the ring. Contrast this to past deflection-based schemes that operated on mesh networks where multiple incoming flits may need to be deflected among a multitude of possible out-bound ports, leading to much more circuit complexity in the router microarchitecture, as shown by [18, 25, 45]. Our application of deflection to rings leads to a simple and elegant embodiment of bufferless routing.

\subsection{Node Router Operation}

At each node on a local ring, we place a single node router, shown in Figure 2. A node router is very simple: it passes through circulating traffic, allows new traffic to enter the ring through a MUX, and allows traffic to leave the ring when it arrives at its destination. Each router contains one pipeline register for the router stage, and one pipeline register for link traversal, so the router latency is exactly one cycle and the per-hop latency is two cycles. Such a design is very common in ring-based and ring-like designs (e.g., [31]).

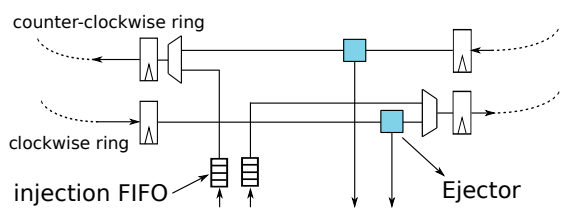

Figure 2: Node router.

As flits enter the router on the ring, they first travel to the ejector. Because we use bidirectional rings, each node router has two ejectors, one per direction 2 Note that the flits constituting a packet may arrive out-of-order and at widely separated times. Re-assembly into packets is thus necessary. Packets are re-assembled and reassembly buffers are managed using the Retransmit-Once scheme, borrowed from the CHIPPER bufferless router design [18]. With this scheme, receivers reassemble packets in-place in MSHRs (Miss-Status Handling Registers [38]), eliminating the need for separate reassembly buffers. The key idea in Retransmit-Once is to avoid ejection backpressureinduced deadlocks by ensuring that all arriving flits are consumed immediately at their

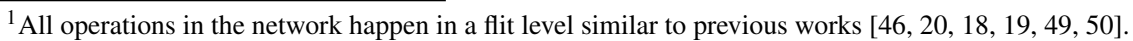

${ }^{2}$ For simplicity, we assume that up to two ejected flits can be accepted by the processor or reassembly buffers in a single cycle. For a fair comparison, we also implement two-flit-per-cycle ejection in our baselines.
} 
receiver nodes. When a flit from a new packet arrives, it allocates a new reassembly buffer slot if available. If no slot is available, the receiver drops the flit and sets a bit in a retransmit queue which corresponds to the sender and transaction ID of the dropped flit. Eventually, when a buffer slot becomes available at the receiver, the receiver reserves the slot for a sender/transaction ID in its retransmit queue and requests a retransmit from the sender. Thus, all traffic arriving at a node is consumed (or dropped) immediately, so ejection never places backpressure on the ring. Retransmit-Once hence avoids protocol-level deadlock [18]. Furthermore, it ensures that a ring full of flits always drains, thus ensuring forward progress (as we will describe more fully in [3]).

After locally-destined traffic is removed from the ring, the remaining traffic travels to the injection stage. At this stage, the router looks for "empty slots," or cycles where no flit is present on the ring, and injects new flits into the ring whenever they are queued for injection. The injector is even simpler than the ejector, because it only needs to find cycles where no flit is present and insert new flits in these slots. Note that we implement two separate injection buffers (FIFOs), one per ring direction; thus, two flits can be injected into the network in a single cycle. A flit enqueues for injection in the direction that yields a shorter traversal toward its destination.

\subsection{Bridge Routers}

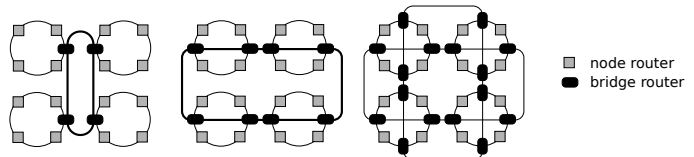

(a) 4-, 8-, and 16-bridge hierarchical ring designs.

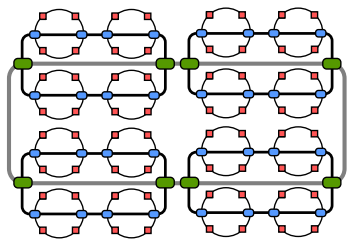

(b) Three-level hierarchy (8x8).

Figure 3: Hierarchical ring design of HiRD.

The bridge routers connect a local ring and a global ring, or a global ring with a higher-level global ring (if there are more than two levels of hierarchy). A high-level block diagram of a bridge router is shown in Figure 4 A bridge router resembles two node routers, one on each of two rings, connected by FIFO buffers in both directions. When a flit arrives on one ring that requires a transfer to the other ring (according to the routing function described below in $\$ 2.3$, it can leave its current ring and wait 
in a FIFO as long as there is space available. These transfer FIFOs exist so that a transferring flit's arrival need not be perfectly aligned with a free slot on the destination ring. However, this transfer FIFO will sometimes fill. In that case, if any flit arrives that requires a transfer, the bridge router simply does not remove the flit from its current ring; the flit will continue to travel around the ring, and will eventually come back to the bridge router, at which point there may be an open slot available in the transfer FIFO. This is analogous to a deflection in hot-potato routing [5], also known as deflection routing, and has been used in recent on-chip mesh interconnect designs to resolve contention [46, 18, 19, 60, 49, 50]. Note that to ensure that flits are eventually delivered, despite any deflections that may occur, we introduce two guarantee mechanisms in [3]. Finally, note that deflections may cause flits to arrive out-of-order (this is fundamental to any non-minimal adaptively-routed network). Because we use Retransmit-Once [18], packet reassembly works despite out-of-order arrival.

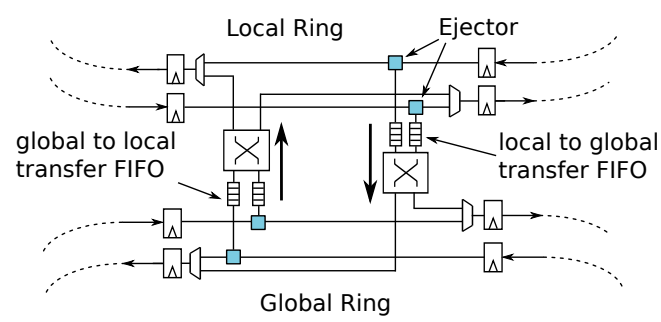

Figure 4: Bridge router.

The bridge router uses crossbars to allow a flit ejecting from either ring direction in a bidirectional ring to enqueue for injection in either direction in the adjoining ring. When a flit transfers, it picks the ring direction that gives a shorter distance, as in a node router. However, these crossbars actually allow for a more general case: the bridge router can actually join several rings together by using larger crossbars. For our network topology, we use hierarchical rings. We use wider global rings than local rings (analogous to a fat tree [26]) for performance reasons. These wider rings perform logically as separate rings as wide as one flit. Although not shown in the figure for simplicity, the bridge router in such a case uses a larger crossbar and has one ring interface (including transfer FIFO) per ring-lane in the wide global ring. The bridge router then load-balances flits between rings when multiple lanes are available. (The 
crossbar and transfer FIFOs are fully modeled in our evaluations.)

When building a two-level design, there are many different arrangements of global rings and bridge routers that can efficiently link the local rings together. Figure $3 \mathrm{a}$ shows three designs denoted by the number of bridge routers in total: 4-bridge, 8bridge, and 16-bridge. We assume an 8-bridge design for the remainder of this paper. Also, note that the hierarchical structure that we propose can be extended to more than two levels. We use a 3-level hierarchy, illustrated in Figure 3b, to build a 64-node network.

Finally, in order to address a potential deadlock case (which will be explained more in [3]), bridge routers implement a special Swap Rule. The Swap Rule states that when the flit that just arrived on each ring requires a transfer to the other ring, the flits can be swapped, bypassing the transfer FIFOs altogether. This requires a bypass datapath (which is fully modeled in our hardware evaluations). It ensures correct operation in the case when transfer FIFOs in both directions are full. Only one swap needs to occur in any given cycle, even when the bridge router connects to a wide global ring. Note that because the swap rule requires this bypass path, the behavior is always active (it would be more difficult to definitively identify a deadlock and enable the behavior only in that special case). The Swap Rule may cause flits to arrive out-of-order when some are bypassed in this way, but the network already delivers flits out-of-order, so correctness is not compromised.

\subsection{Routing}

Finally, we briefly address routing. Because a hierarchical ring design is fundamentally a tree, routing is very simple: when a flit is destined for a node in another part of the hierarchy, it first travels up the tree (to more global levels) until it reaches a common ancestor of its source and its destination, and then it travels down the tree to its destination. Concretely, each node's address can be written as a series of parts, or digits, corresponding to each level of the hierarchy (these trivially could be bitfields in a node ID). A ring can be identified by the common prefix of all routers on that ring; the root global ring has a null (empty) prefix, and local rings have prefixes consisting of all digits but the last one. If a flit's destination does not match the prefix of the ring it 
is on, it takes any bridge router to a more global ring. If a flit's destination does match the prefix of the ring it is on (meaning that it is traveling down to more local levels), it takes any bridge router which connects to the next level, until it finally reaches the local ring of its destination and ejects at the node with a full address match.

\subsection{Guaranteed Delivery: Correctness in Hierarchical Ring Interconnects}

We provide a rigorous description of how our mechanism guarantees packet delivery in our original work available in Section III of [3]. We also show that the required hardware cost is minimal in Section III.D of the same work. We refer the reader to the original work for these analyses [3].

\section{Evaluation Methodology}

We perform our evaluations using a cycle-accurate simulator of a CMP system with $1.6 \mathrm{GHz}$ interconnect to provide application-level performance results [52]. Our simulator is publicly available and includes the source code of all mechanisms we evaluated [52]. Tables 1 and 2 provide the configuration parameters of our simulated systems.

\begin{tabular}{|l||l|}
\hline Parameter & Setting \\
\hline \hline System topology & CPU core and shared cache slice at every node \\
\hline Core model & $\begin{array}{l}\text { Out-of-order, 128-entry ROB, 16 MSHRs (maximum simultaneous outstanding re- } \\
\text { quests) }\end{array}$ \\
\hline Private L1 cache & 64 KB, 4-way associative, 32-byte block size \\
\hline Shared L2 cache & $\begin{array}{l}\text { Perfect (always hits) to stress the network and penalize our reduced-capacity } \\
\text { deflection-based design; cache-block-interleaved }\end{array}$ \\
\hline Cache coherence & $\begin{array}{l}\text { Directory-based protocol (based on SGI Origin [41]), directory entries co-located } \\
\text { with shared cache blocks }\end{array}$ \\
\hline Simulation length & 5M-instruction warm-up, 25M-instruction active execution per node [46 18 \\
\hline
\end{tabular}

Table 1: Simulation and system configuration parameters.

Our methodology ensures a rigorous and isolated evaluation of NoC capacity for especially cache-resident workloads, and has also been used in other studies [46, 18, 49, 50, 19]. Instruction traces for the simulator are taken using a Pintool [44] on representative portions of SPEC CPU2006 workloads.

We mainly compare to a single bidirectional ring and a state-of-the-art buffered hierarchical ring [53]. Also, note that while there are many possible ways to optimize 


\begin{tabular}{|c|c|c|}
\hline Parameter & Network & Setting \\
\hline \multirow{3}{*}{ Interconnect Links } & Single Ring & $\begin{array}{l}\text { Bidirectional, 4x4: } 64 \text {-bit and 128-bit width, 8x8: } 128 \text {-bit } \\
\text { and 256-bit width }\end{array}$ \\
\hline & Buffered HRing & $\begin{array}{l}\text { Bidirectional, 4x4: } 3 \text {-cycle per-hop latency (link+router); } \\
64 \text {-bit local and 128-bit global rings, } 8 \times 8 \text { : three-level hier- } \\
\text { archy, } 4 \times 4 \text { parameters, with second-level rings connected } \\
\text { by a } 256 \text {-bit third-level ring }\end{array}$ \\
\hline & HiRD & $\begin{array}{l}\text { 4x4: 2-cycle (local), 3-cycle (global) per-hop latency } \\
\text { (link+router); 64-bit local ring, 128-bit global ring; } 8 \times 8 \text { : } \\
\text { 4x4 parameters, with second-level rings connected by a } \\
\text { 256-bit third-level ring }\end{array}$ \\
\hline \multirow{3}{*}{ Router } & Single Ring & 1-cycle per-hop latency (as in [33]) \\
\hline & Buffered HRing & $\begin{array}{l}\text { Node (NIC) and bridge (IRI) routers based on }[53] \text {; 4-flit } \\
\text { in-ring and transfer FIFOs. Bidirectional links of dual-flit } \\
\text { width (for fair comparison with our design). Bubble flow } \\
\text { control [7] for deadlock freedom. }\end{array}$ \\
\hline & HiRD & $\begin{array}{l}\text { Local-to-global buffer depth of } 1 \text {, global-to-local buffer } \\
\text { depth of } 4\end{array}$ \\
\hline
\end{tabular}

Table 2: Network parameters.

each baseline (such as congestion control [8, 49, 50], adaptive routing schemes, and careful parameter tuning), we assume a fairly typical aggressive configuration for each.

Data Mapping: We map data in a cache-block-interleaved way to different shared L2 cache slices. This mapping is agnostic to the underlying locality. As a result, it does not exploit the low-latency data access in the local ring. One can design systematically better mapping in order to keep frequently used data in the local ring as in [42, 10]. However, such a mapping mechanism is orthogonal to our proposal and can be applied in all ring-based network designs.

Application \& Synthetic Workloads: The system is run with a set of 60 multiprogrammed workloads. Each workload consists of one single-threaded instance of a SPEC CPU2006 benchmark on each core, for a total of either $16(4 \times 4)$ or $64(8 \times 8)$ benchmark instances per workload. Multiprogrammed workloads such as these are representative of many common workloads for large CMPs. Workloads are constructed at varying network intensities as follows: first, benchmarks are split into three classes (Low, Medium and High) by L1 cache miss intensity (which correlates directly with network injection rate), such that benchmarks with less than 5 misses per thousand instructions (MPKI) are "Low-intensity," between 5 and 50 are "Medium-intensity," and above 50 MPKI are "High-intensity." Workloads are then constructed by randomly selecting a certain number of benchmarks from each category. We form workload sets with four intensity mixes: High (H), Medium (M), Medium-Low (ML), and Low (L), 
with 15 workloads in each (the average network injection rates for each category are $0.47,0.32,0.18$, and 0.03 flits/node/cycle, respectively).

Multithreaded Workloads: We use the GraphChi implementation of the GraphLab framework [40, 43]. The implementation we use is designed to run efficiently on multicore systems. The workload consists of Twitter Community Detection (CD), Twitter Page Rank (PR), Twitter Connected Components (CC), Twitter Triangle Counting (TC) [39], and Graph500 Breadth First Search (BFS). We simulated the representative portion of each workload and each workload has a working set size of greater than 151.3 MB. On every simulation of these multithreaded workloads, we warm up the cache with the first 5 million instructions, then we run the remaining code of the representative portion.

Energy \& Area: We measure the energy and area of routers and links by individually modeling the crossbar, pipeline registers, buffers, control logic, and other datapath components. For links, buffers and datapath elements, we use DSENT 0.91 [58]. Control logic is modeled in Verilog RTL. Both energy and area are calculated based on a $45 \mathrm{~nm}$ technology. The link lengths we assume are based on the floorplan of our designs, which we describe in the next paragraph.

We assume the area of each core to be $2.5 \mathrm{~mm} \times 2.5 \mathrm{~mm}$. We assume a $2.5 \mathrm{~mm}$ link length for single-ring designs. For the hierarchical ring design, we assume $1 \mathrm{~mm}$ links between local-ring routers, because the four routers on a local ring can be placed at four corners that meet in a tiled design. Global-ring links are assumed to be $5.0 \mathrm{~mm}$ (i.e., five times as long as local links), because they span across two tiles on average if local rings are placed in the center of each four-tile quadrant. Third-level global ring links are assumed to be $10 \mathrm{~mm}$ (i.e., ten times as long as local links) in the $8 \times 8$ evaluations. This floorplan is illustrated in more detail in Figure 5 for the 3-level (64node) HiRD network. Note that one quadrant of the floorplan of Figure 5 corresponds to the floorplan of the 2-level (16-node) HiRD network. We faithfully take into account all link lengths in our energy and area estimates for all designs.

Application Evaluation Metrics: For multiprogrammed workloads, we present application performance results using the commonly-used Weighted Speedup metric [57, 17]. We use the maximum slowdown metric to measure unfairness [14, 34, 35, 62, 16, 


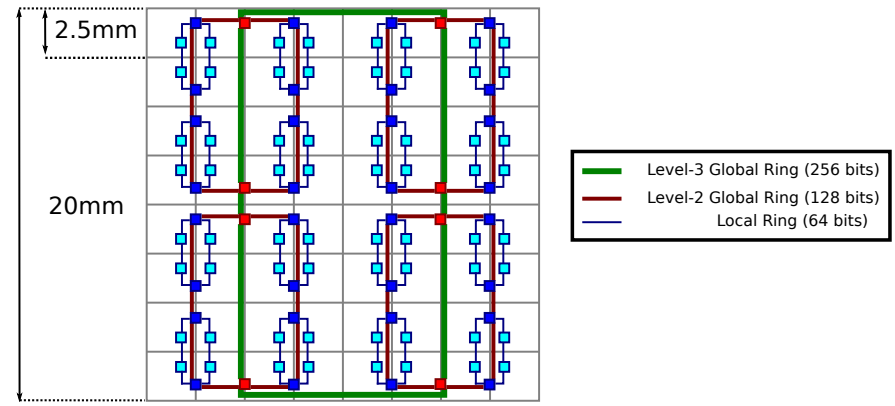

Figure 5: Assumed floorplan for HiRD 3-level (64-node) network. Two-level (16-node) network consists of one quadrant of this floorplan.

48].

\section{Evaluation}

We provide a comprehensive evaluation of our proposed mechanism against other ring baselines. Since our goal is to provide a better ring design, our main comparisons are to ring networks. However, we also provide sensitivity analyses and comparisons to other network designs as well.

\subsection{Ring-based Network Designs}

\section{Multiprogrammed workloads}

Figure 6 6 shows performance (weighted speedup normalized per node), power (total network power normalized per node), and energy-efficiency (perf./power) for 16-node and 64-node HiRD and buffered hierarchical rings in [53], using identical topologies, as well as a single ring (with different bisection bandwidths).

1. A hierarchical topology yields significant performance advantages over a single ring (i) when network load is high and/or (ii) when the network scales to many nodes. As shown, the buffered hierarchical ring improves performance by 7\% (and HiRD by $10 \%$ ) in high-load workloads at 16 nodes compared to a single ring with 128-bit links. The hierarchical design also reduces power because hop count is reduced. Therefore, link power reduces significantly with respect to a single ring. On average, in the $8 \times 8$ configuration, the buffered hierarchical ring network obtains $15.6 \%$ better application 


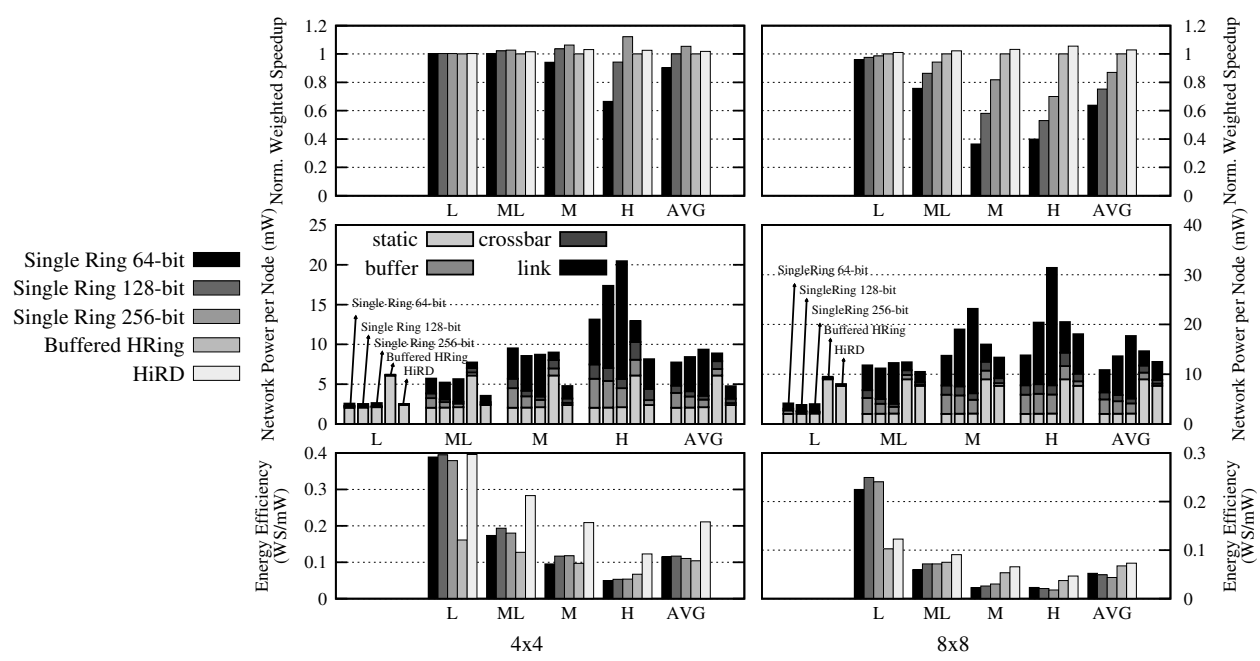

Figure 6: HiRD as compared to buffered hierarchical rings and a single-ring network.

performance than the single ring with 256-bit links, while HiRD attains $18.2 \%$ higher performance.

2. Compared to the buffered hierarchical ring, HiRD has significantly lower network power and better performance. On average, HiRD reduces total network power (links and routers) by $46.5 \%(4 \times 4)$ and $14.7 \%(8 \times 8)$ relative to this baseline. This reduction in turn yields significantly better energy efficiency (lower energy consumption for buffers and slightly higher for links). ${ }^{3}$ Overall, HiRD is the most energy-efficient of the ring-based designs evaluated in this paper for both $4 \times 4$ and $8 \times 8$ network sizes. HiRD also performs better than Buffered HRing due to the reasons explained in the next section $(\$ 4.2)$.

3. While scaling the link bandwidth increases the performance of a single ring network, the network power increases $25.9 \%$ when the link bandwidth increases from 64-bit to 128 -bit and $15.7 \%$ when the link bandwidth increases from 128-bit to 256-bit because of higher dynamic energy due to wider links. In addition, scaling the link bandwidth is not a scalable solution as a single ring network performs worse than the bufferred hierarchical ring baseline even when a 256-bit link is used.

\footnotetext{
${ }^{3}$ Note that the low intensity workloads in the $8 \times 8$ network is an exception. HiRD reduces energy efficiency for these as the static link power becomes dominant for them.
} 


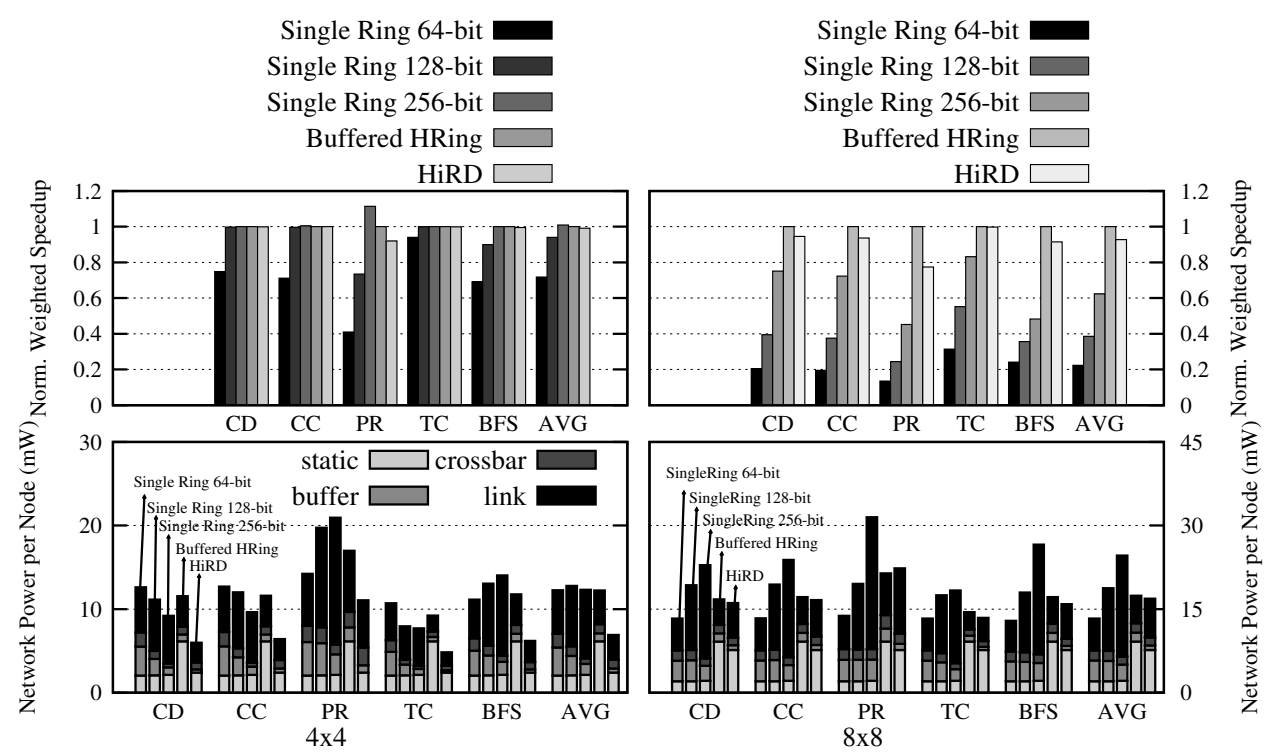

Figure 7: HiRD as compared to buffered hierarchical rings and a single-ring network on multithreaded workloads.

We conclude that HiRD is effective in simplifying the design of the hierarchical ring and making it more energy efficient, as we intended to as our design goal. We show that HiRD provides competitive performance compared to the baseline buffered hierarchical ring design with equal or better energy efficiency.

\section{Multithreaded workloads}

Figure 7 shows the performance and power of HiRD on multithreaded applications compared to a buffered hierarchical ring and a single-ring network for both 16-node and 64 -node systems. On average, HiRD performs $0.1 \%(4 \times 4)$ and $0.73 \%(8 \times 8)$ worse than the buffered hierarchical ring. However, on average, HiRD consumes $43.8 \%(4 \mathrm{x} 4)$ and $3.1 \%(8 \times 8)$ less power, leading to higher energy efficiency. This large reduction in energy comes from the elimination of most buffers in HiRD.

Both the buffered hierarchical ring and HiRD outperform single ring networks, and the performance improvement increases as we scale the size of the network.

Even though HiRD performs competitively with a buffered hierarchical ring network in most cases, HiRD performs poorly on the Page Ranking application. We observe that Page Ranking generates more non-local network traffic than other applications. As HiRD is beneficial mainly at lowering the local-ring latency, it is unable 
to speed up such non-local traffic, and is thus unable to help Page Ranking. In addition, Page Ranking also has higher network traffic, causing more congestion in the network (we observe $17.3 \%$ higher average network latency for HiRD in an 8x8 network), and resulting in a performance drop for HiRD. However, it is possible to use a different number of bridge routers as illustrated in Figure $3 \mathrm{a}$. to improve the performance of HiRD, which we will analyze in Section 4.8. Additionally, it is possible to apply a locality-aware cache mapping technique [42, 10] in order to take advantage of lower local-ring latency in HiRD.

We conclude that HiRD is effective in improving evergy efficiency significantly for both multiprogrammed and multithreaded applications.

\subsection{Synthetic-Traffic Network Behavior}

Figure 8 shows the average packet latency as a function of injection rate for buffered and bufferless mesh routers, a single-ring design, the buffered hierarchical ring, and HiRD in 16 and 64-node systems. We show uniform random, transpose and bit complement traffic patterns [11]. Sweeps on injection rate terminate at network saturation. The buffered hierarchical ring saturates at a similar point to HiRD but maintains a slightly lower average latency because it avoids transfer deflections. In contrast to these high-capacity designs, the 256-bit single ring saturates at a lower injection rate.

As network size scales to $8 \times 8$, HiRD performs significantly better than the 256 bit single ring, because the hierarchy reduces the cross-chip latency while preserving bisection bandwidth. HiRD also performs better than Buffered HRing because of two reasons. First, HiRD is able to allow higher peak utilization $(91 \%)$ than Buffered HRing $(71 \%)$ on the global rings. We observed that when flits have equal distance in a clock-wise and counter clock-wise direction, Buffered HRing has to send flits to one direction in order to avoid deadlock while deflections in HiRD allow flits to travel in both directions, leading to better overall network utilization. Second, at high injection rates, the transfer guarantee [3] starts throttling the network, disallowing future flits to be injected into the network until the existing flits arrive at their destinations. This reduces congestion in the network and allows HiRD to saturate at a higher injection rate than the buffered hierarchical ring design. 

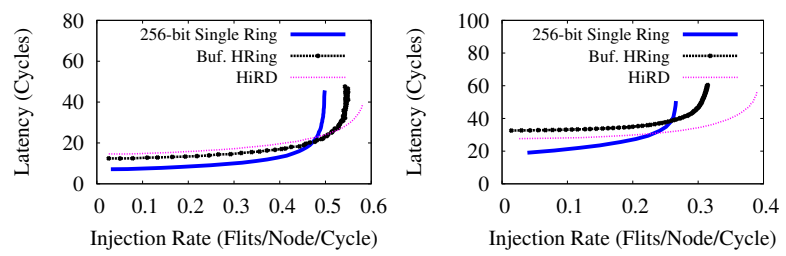

(a) Uniform Random, $4 \times 4$

(b) Uniform Random, $8 \times 8$
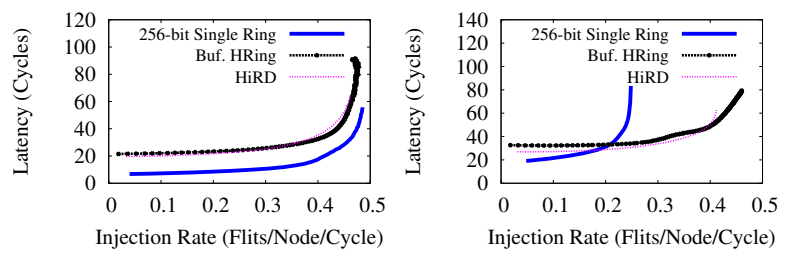

(c) Bit Complement, $4 \times 4$

$$
\text { (d) Bit Complement, } 8 \times 8
$$
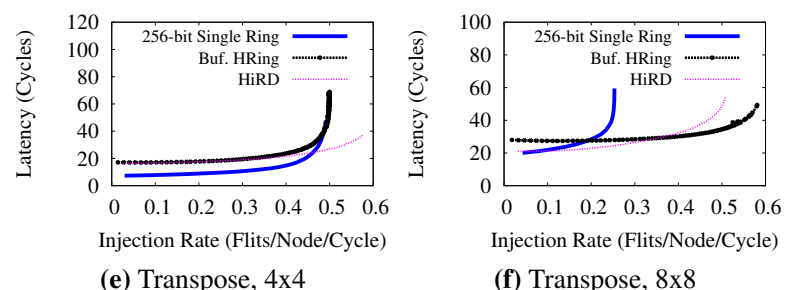

(e) Transpose, $4 \times 4$

(f) Transpose, $8 \times 8$

Figure 8: Synthetic-traffic evaluations for $4 \times 4$ and $8 \times 8$ networks.

\subsection{Injection and Transfer Guarantees}

In this subsection, we study HiRD's behavior under a worst-case synthetic traffic pattern that triggers the injection and transfer guarantees and demonstrates that they are necessary for correct operation, and that they work as designed.

Traffic Pattern: In the worst-case traffic pattern, all nodes on three rings in a twolevel (16-node) hierarchy inject traffic (we call these rings Ring A, Ring B, and Ring C). Rings A, B, and C have bridge routers adjacent to each other, in that order, on the single global ring. All nodes in Ring A continuously inject flits to nodes in Ring C, and all nodes in Ring $\mathrm{C}$ likewise inject flits to nodes in Ring A. This creates heavy traffic on the global ring across the point at which Ring B's bridge router connects. All nodes on Ring B continuously inject flits (whenever they are able) addressed to another ring elsewhere in the network. However, because Rings A and C continuously inject flits, Ring B's bridge router will not be able to transfer any flits to the global ring in the steady state (unless another mechanism such as the throttling mechanism in [3] 


\begin{tabular}{|l||l|l|l||l|l|}
\hline \multirow{2}{*}{ Configuration } & \multicolumn{3}{|c||}{$\begin{array}{c}\text { Network Throughput } \\
\text { (flits/node/cycle) }\end{array}$} & $\begin{array}{c}\text { Transfer FIFO Wait } \\
\text { (cycles) }\end{array}$ & Deflections/Retries \\
\hline & Ring A & Ring B & Ring C & avg/max & avg/max \\
\hline \hline Without Guarantees & 0.164 & 0.000 & 0.163 & $2.5 / 299670$ & $6.0 / 49983$ \\
\hline With Guarantees & 0.133 & 0.084 & 0.121 & $1.2 / 66$ & $2.8 / 18$ \\
\hline
\end{tabular}

Table 3: Results of worst-case traffic pattern without and with injection/transfer guarantees enabled.

intercedes).

Results: Table 3 shows three pertinent metrics on the network running the described traffic pattern: average network throughput (flits/node/cycle) for nodes on Rings A, B, and $\mathrm{C}$, the maximum time (in cycles) spent by any one flit at the head of a transfer FIFO, and the maximum number of times any flit is deflected and has to circle a ring to try again. These metrics are reported with the injection and transfer guarantee mechanisms disabled and enabled. The experiment is run with the synthetic traffic pattern for $300 \mathrm{~K}$ cycles.

The results show that without the injection and transfer guarantees, Ring B is completely starved and cannot transfer any flits onto the global ring. This is confirmed by the maximum transfer FIFO wait time, which is almost the entire length of the simulation. In other words, once steady state is reached, no flit ever transfers out of Ring B. Once the transfer FIFO in Ring B's bridge router fills, the local ring fills with more flits awaiting a transfer, and these flits are continuously deflected. Hence, the maximum deflection count is very high. Without the injection or transfer guarantees, the network does not ensure forward progress for these flits. In contrast, when the injection and transfer guarantees are enabled, (i) Ring B's bridge router is able to inject flits into the global ring and (ii) Ring B's bridge router fairly picks flits from its local ring to place into its transfer FIFO. The maximum transfer FIFO wait time and maximum deflection count are now bounded, and nodes on all rings receive network throughput. Thus, the guarantees are both necessary and sufficient to ensure deterministic forward progress for all flits in the network.

Real Applications: Table 4 shows the effect of the transfer guarantee mechanism on real applications in a $4 \times 4$ network. Average transfer FIFO wait time shows the average number of cycles that a flit waits in the transfer FIFO across all 60 workloads. Maximum transfer FIFO wait time shows the maximum observed flit wait time in the same 


\begin{tabular}{|l||l|l|}
\hline Configuration & Transfer FIFO Wait time (cycles) & Deflections/Retries \\
\hline & $(\mathrm{avg} / \mathrm{max})$ & $(\mathrm{avg} / \mathrm{max})$ \\
\hline \hline Without guarantees & $3.3 / 169$ & $3.7 / 19$ \\
\hline With guarantees & $0.76 / 72$ & $0.7 / 8$ \\
\hline
\end{tabular}

Table 4: Effect of transfer guarantee mechanism on real workloads.

FIFO across all workloads. As illustrated in Table 4, some number of flits can experience very high wait times when there is no transfer guarantee. Our transfer guarantee mechanism reduces both average and maximum FIFO wait times 4 In addition, we observe that our transfer guarantee mechanism not only provides livelock- and deadlockfreedom but also provides lower maximum wait time in the transfer FIFO for each flit because the guarantee provides a form of throttling when the network is congested. A similar observation has been made in many previous network-on-chip works that use source throttling to improve the performance of the network [59, 6, 49, 8, 50].

We conclude that our transfer guarantee mechanism is effective in eliminating livelock and deadlock as well as reducing packet queuing delays in real workloads.

\subsection{Network Latency and Latency Distribution}

Figure 9 shows average network latency for our three evaluated configurations: 256-bit single ring, buffered hierarchical ring and HiRD. This plot shows that our proposal can reduce the network latency by having a faster local-ring hop latency compared to other ring-based designs. Additionally, we found that, for all real workloads, the number of deflections we observed is always less than $3 \%$ of the total number of flits. Therefore, the benefit of our deflection based router design outweighs the extra cost of deflections compared to other ring-based router designs. Finally, in the case of small networks such as a $4 \times 4$ network, a 1-cycle hop latency of a single ring provides significant latency reduction compared to the buffered hierarchical design. However, a faster local-ring hop latency in HiRD helps to reduce the network latency of a hierarchical design and provides a competitive network latency compared to a single ring design in small networks.

\footnotetext{
${ }^{4}$ As the network scales to 64 nodes, we observe that the average wait time in the transfer FIFO does not affect the overall performance significantly (adding 1.5 cycles per flit).
} 

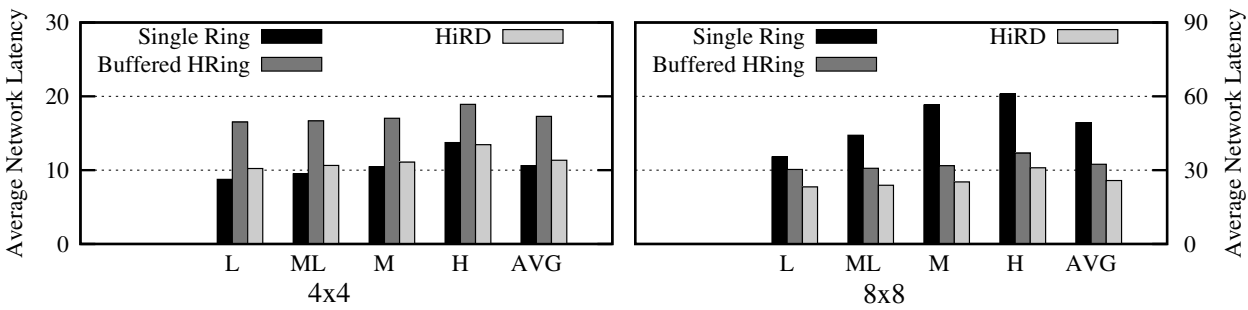

Figure 9: Average network latency for $4 \times 4$ and $8 \times 8$ networks.

In addition, Figure 10 shows the maximum latency and Figure 11 shows the 95th percentile latency for each network design. The 95th percentile latency shows the behavior of the network without extreme outliers. These two figures provide quantitative evidence that the network is deadlock-free and livelock-free. Several observations are in order:
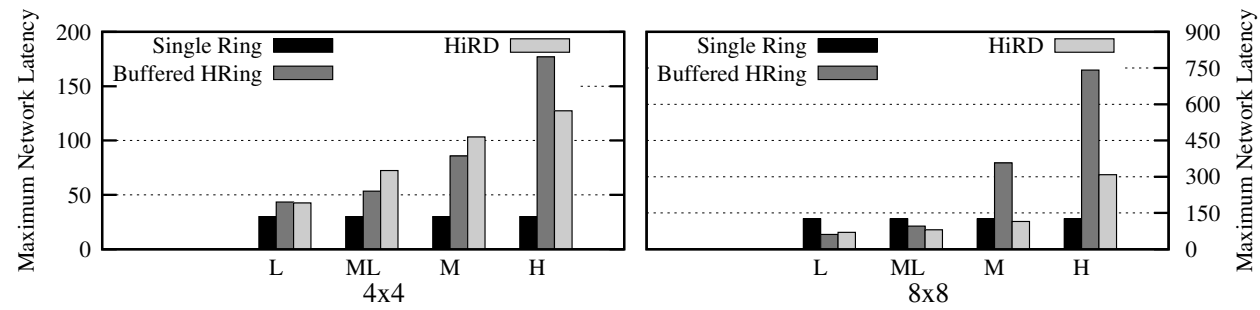

Figure 10: Maximum network latency for $4 \times 4$ and $8 \times 8$ networks.
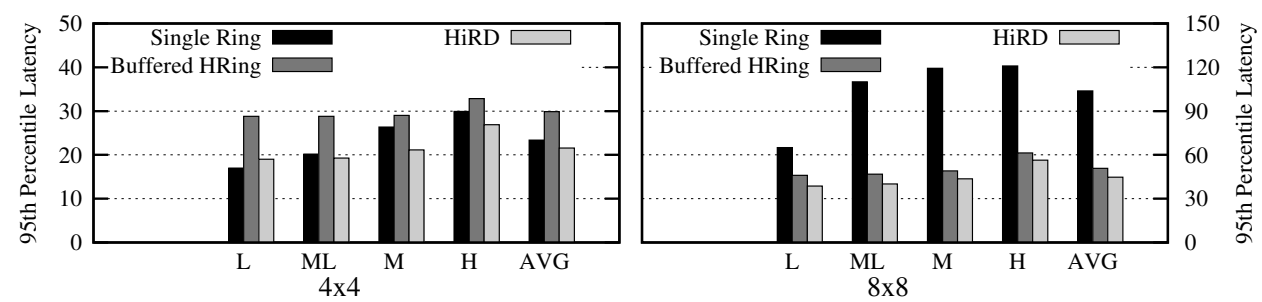

Figure 11: 95th percentile latency for $4 \times 4$ and $8 \times 8$ networks.

1. HiRD provides lower latency at the 95th percentile and the lowest average latency observed in the network. This lower latency comes from our transfer guarantee mechanism, which is triggered when flits spend more than 100 cycles in each local ring, draining all flits in the network to their destination. This also means that HiRD improves the worst-case latency that a flit can experience because none of the flits are severely delayed. 
2. While both HiRD and the buffered hierarchical ring have higher 95th percentile and maximum flit latency compared to a 64-bit single ring network, both hierarchical designs have $60.1 \%$ (buffered hierarchical ring) and 53.9\% (HiRD) lower average network latency in an $8 \times 8$ network because a hierarchical design provides better scalability on average.

3. Maximum latency in the single ring is low because contention happens only at injection and ejection, as opposed to hierarchical designs where contention can also happen when flits travel through different level of the hierarchy.

4. The transfer guarantee in HiRD also helps to significantly reduce the maximum latency observed by some flits compared to a buffered design because the guarantee enables the throttling of the network, thereby alleviating congestion. Reduced congestion leads to reduced maximum latency. This observation is confirmed by our synthetic traffic results shown in Section 4.2

\subsection{Fairness}

Figure 12 shows the fairness, measured by the maximum slowdown metric, for our three evaluated configurations. Compared to a buffered hierarchical ring design HiRD, is $8.3 \%(5.1 \%)$ more fair on a $4 \times 4(8 \times 8)$ network. Compared to a single ring design, HiRD is $40.0 \%(296.4 \%)$ more fair on a $4 \times 4(8 \times 8)$ network. In addition, we provide several observations:
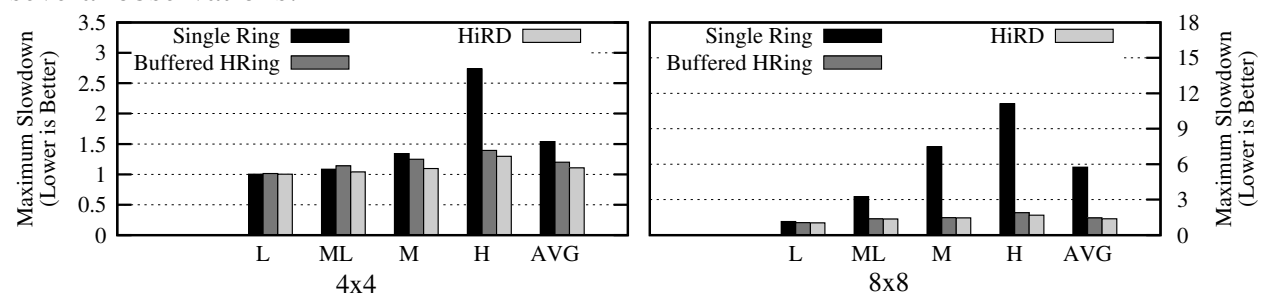

Figure 12: Unfairness for $4 \times 4$ and $8 \times 8$ networks.

1. HiRD is the most fair design compared to the buffered hierarchical ring and the single ring designs. Compared to a single ring design, hierarchical designs are more fair because the global ring in the hierarchical designs allows flits to arrive at the destination faster. Compared to the buffered hierarchical ring design, HiRD is more fair because HiRD has lower average network latency. HiRD is much more fair for medium 
and high intensity workloads, where the throttling mechanism in HiRD lowers average network latency.

2. Global rings allow both hierarchical designs to provide better fairness compared to the single ring design as the size of the network gets bigger from $4 \times 4$ to $8 \times 8$.

3. We conclude that HiRD is the most fair ring design among all evaluated designs due to its overall lower packet latencies and reduced congestion across all applications.

\subsection{Router Area and Timing}

We show both critical path length and normalized die area for single-ring, buffered hierarchical ring, and HiRD, in Table 5 . Area results are normalized to the buffered hierarchical ring baseline, and are reported for all routers required by a 16-node network (e.g., for HiRD, 16 node routers and 8 bridge routers).

\begin{tabular}{|l||l|l|l|}
\hline Metric & Single-Ring & Buffered HRing & HiRD \\
\hline \hline Critical path (ns) & 0.33 & 0.87 & 0.61 \\
\hline Normalized area & 0.281 & 1 & 0.497 \\
\hline
\end{tabular}

Table 5: Total router area (16-node network) and critical path.

Two observations are in order. First, HiRD reduces area relative to the buffered hierarchical ring routers, because the node router required at each network node is much simpler and does not require complex flow control logic. HiRD reduces total router area by $50.3 \%$ vs. the buffered hierarchical ring. Its area is higher than a single ring router because it contains buffers in bridge routers. However, the energy efficiency of HiRD and its performance at high load make up for this shortcoming. Second, the buffered hierarchical ring router's critical path is $42.6 \%$ longer than HiRD because its control logic must also handle flow control (it must check whether credits are available for a downstream buffer). The single-ring network has a higher operating frequency than HiRD because it does not need to accommodate ring transfers (but recall that this simplicity comes at the cost of poor performance at high load for the single ring).

\subsection{Sensitivity to Link Bandwidth}

The bandwidth of each link also has an effect on the performance of different network designs. We evaluate the effect of different link bandwidths on several ring-based networks by using 32-, 64- and 128-bit links on all network designs. Figure 13 shows the performance and power consumption of each network design. As links get wider, 
the performance of each design increases. According to the evaluation results, HiRD performs slightly better than a buffered hierarchical ring design for almost all link bandwidths while maintaining much lower power consumption on a $4 \times 4$ network, and slightly lower power consumption on an $8 \times 8$ network.
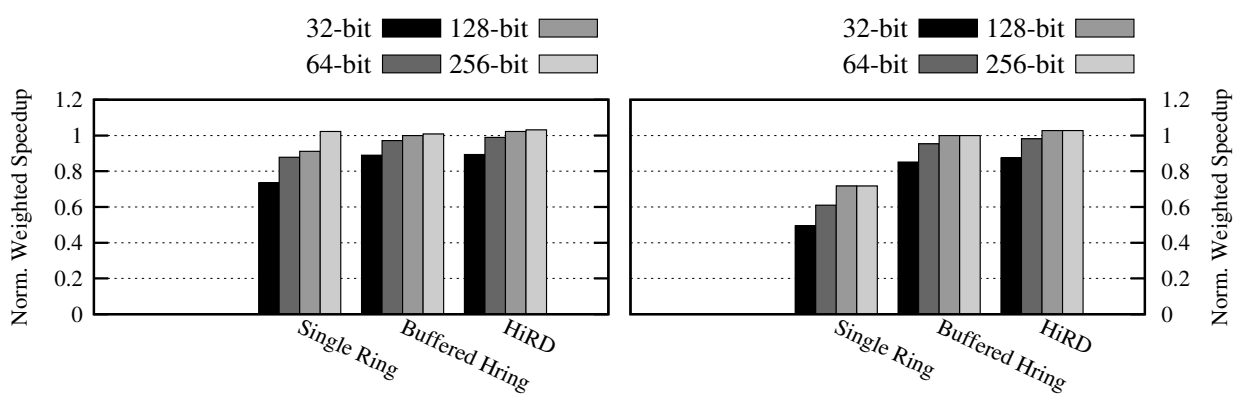

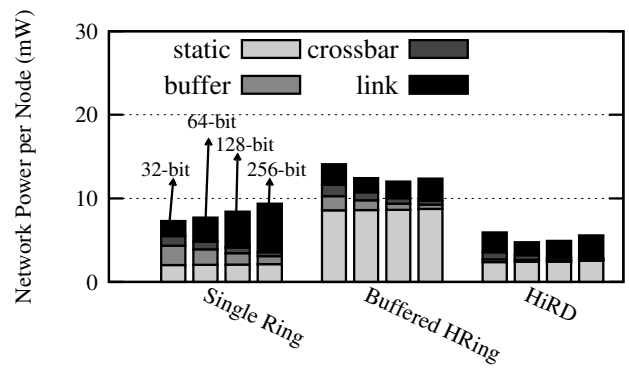

$4 \times 4$

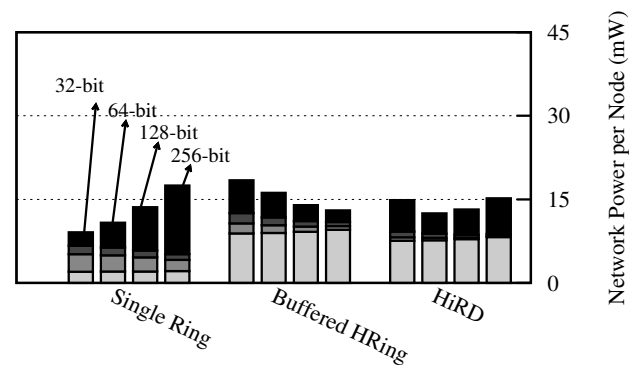

$8 \times 8$

Figure 13: Sensitivity to different link bandwidth for $4 \times 4$ and $8 \times 8$ networks.

Additionally, we observe that increasing link bandwidth can decrease the network power in a hierarchical design because lower link bandwidth causes more congestion in the network and leads to more dynamic buffer, crossbar and link power consumption due to additional deflections at the buffers. As the link bandwidth increases, congestion reduces, lowering dynamic power. However, we observe that past a certain link bandwidth (e.g., 128 bits for buffered hierarchical ring and HiRD), congestion no longer reduces, because deflections at the buffers become the bottleneck instead. This leads to diminishing returns in performance yet increased dynamic power.

\subsection{Sensitivity to Configuration Parameters}

Bridge Router Organization: The number of bridge routers connecting the global ring(s) to the local rings has an important effect on system performance because the 
connection between local and global rings can limit bisection bandwidth. In Figure $3 \mathrm{a}$, we showed three alternative arrangements for a 16-node network, with 4, 8, and 16 bridge routers. So far, we have assumed an 8-bridge design in 4x4-node systems, and a system with 8 bridge routers at each level in $8 \times 8$-node networks (Figure 3b). In Figure 14a. we show average performance across all workloads for a $4 \mathrm{x} 4$-node system with 4,8 , and 16 bridge routers. Buffer capacity is held constant. As shown, significant performance is lost if only 4 bridge routers are used (10.4\% on average). However, doubling from 8 to 16 bridge routers gains only $1.4 \%$ performance on average. Thus, the 8-bridge design provides the best tradeoff of performance and network cost (power and area) overall in our evaluations.
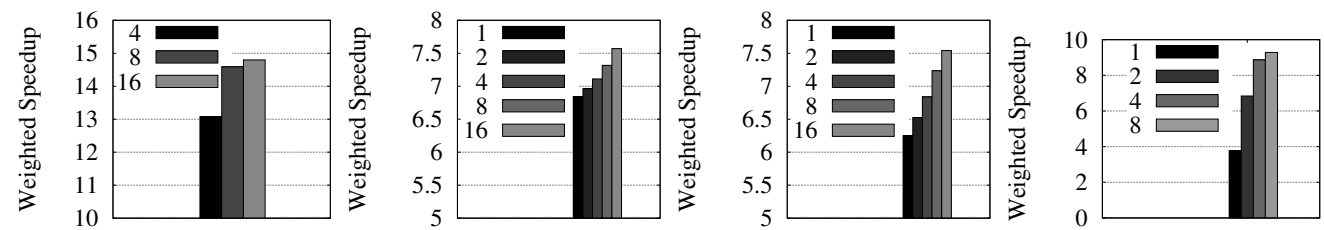

(a) Number of bridge routers

(b) Local-to-global bridge buffer (c) Global-to-local bridge buffer (d) Global-Local ring B/W ratio

Figure 14: Performance sensitivity to buffer sizes and the global ring bandwidth in a $4 \times 4$ network.

Bridge Router Buffer Size: The size of the FIFO queues used to transfer flits between local and global rings can have an impact on performance if they are too small (and hence are often full, leading them to deflect transferring flits) or too large (and hence increase bridge router power and die area). We show the effect of local-to-global and global-to-local FIFO sizes in Figures $14 \mathrm{~b}$ and $14 \mathrm{c}$ respectively, for the 8-bridge $4 \times 4$-node design. In both cases, increased buffer size leads to increased performance. However, performance is more sensitive to global-to-local buffer size (20.7\% gain from 1-flit to 16-flit buffer size) than to local-to-global size (10.7\% performance gain from 1 to 16 flits), because in the 8-bridge configuration, the whole-loop latency around the global ring is slightly higher than the loop latency in each of the local ring, making a global-to-local transfer retry more expensive than a local-to-global one.

For our evaluations, we use a 4-flit global-to-local and 1-flit local-to-global buffer per bridge router, which results in transfer deflection rates of $28.2 \%$ (global-to-local) and $34 \%$ (local-to-global) on average for multiprogrammed workloads. These deflec- 
tion rates are less than $1 \%$ for all of our multithreaded workloads. The deflection rate is much lower in multithreaded workloads because these workloads are less memoryintensive and hence the contention in the on-chip interconnect is low for them.

Global Ring Bandwidth: Previous work on hierarchical ring designs does not examine the impact of global ring bandwidth on performance but instead assume equal bandwidth in local and global rings [54]. In Figure 14d, we examine the sensitivity of system performance to global ring bandwidth relative to local ring bandwidth, for the all-High category of workloads (in order to stress check bisection bandwidth). Each point in the plot is described by this global-to-local ring bandwidth ratio. The local ring design is held constant while the width of the global ring is adjusted. If a ratio of $1: 1$ is assumed (leftmost bar), performance is significantly worse than the best possible design. Our main evaluations in 4x4 networks use a ratio of 2:1 (global:local) in order to provide equivalent bisection bandwidth to a $4 \times 4$ mesh baseline. Performance increases by $81.3 \%$ from a 1:1 ratio to the $2: 1$ ratio that we use. After a certain point, the global ring becomes less of a bottleneck, and further global-ring bandwidth increases have massively smaller effects.

Delivery Guarantee Parameters: We introduced injection guarantee and ejection guarantee mechanisms to ensure every flit is eventually delivered to its destination. These guarantees are clearly described in detail in our original work [3]. The injection guarantee mechanism takes a threshold parameter that specifies how long an injection can be blocked before action is taken. Setting this parameter too low can have an adverse impact on performance, because the system throttles nodes too aggressively and thus underutilizes the network. Our main evaluations use a 100-cycle threshold. For high-intensity workloads, performance drops by $21.3 \%$ when using an aggressive threshold of only 1 cycle. From 10 cycles upward, variation in performance is at most $0.6 \%$ : the mechanism is invoked rarely enough that the exact threshold does not matter, only that it is finite (is required for correctness guarantees). In fact, for a 100-cycle threshold, the injection guarantee mechanism is never triggered in our real applications. Hence, the mechanism is necessary only for corner-case correctness. In addition, we evaluate the impact of communication latency between routers and the coordinator. We find less than $0.1 \%$ variation in performance for latencies ranging from 1 to 30 cycles 
(when parameters are set so that the mechanism becomes active); thus, slow, low-cost wires may be used for this mechanism.

The ejection guarantee takes a single threshold parameter: the number of times a flit is allowed to circle around a ring before action is taken. We find less than $0.4 \%$ variation in performance when sweeping the threshold from 1 to 16 . Thus, the mechanism provides correctness in corner cases but is unimportant for performance in the common case.

\subsection{Comparison Against Other Ring Configurations}

Figure 15 highlights the energy-efficiency comparison of different ring-based design configurations by showing weighted speedup ( $\mathrm{Y}$ axis) against power ( $\mathrm{X}$ axis) for all evaluated $4 \times 4$ networks. HiRD is shown with the three different bridge-router configurations (described in $\$ 2.2$. Every ring design is evaluated at various link bandwidths (32-, 64-, 128- and 256-bit links). The top-left is the ideal corner (high performance, low power). As the results show, at the same link bandwidth, all three configurations of HiRD are more energy efficient than the evaluated buffered hierarchical ring baseline designs at this network size.

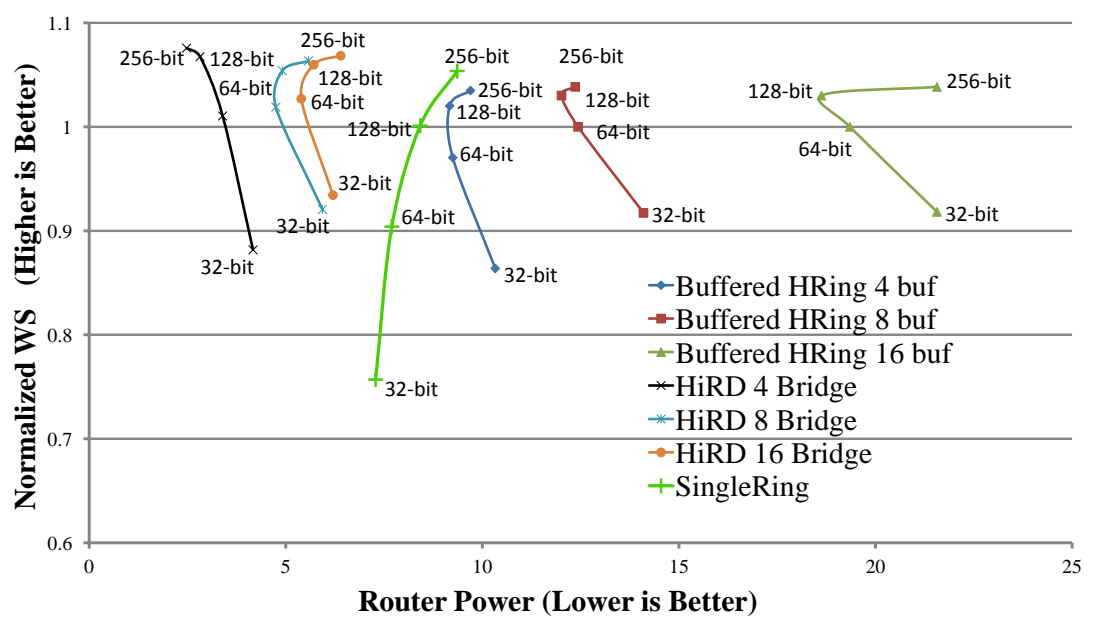

Figure 15: Weighted speedup (Y) vs. power (X) for $4 \times 4$ networks.

We also observe that increasing link bandwidth can sometimes decrease router power as it reduces deflections in HiRD or lowers contention at the buffers in a buffered hierarchical ring design. However, once links are wide enough, this benefit diminishes 
for two reasons: 1) links and crossbars consume more energy, 2) packets arrive at the destination faster, leading to higher power as more energy is consumed in less time.

\subsection{Comparison Against Other Network Designs}

For completeness, Table 6 compares HiRD against several other network designs on $4 \times 4$ and $8 \times 8$ networks using the multiprogrammed workloads described in Section 3 .

We compare our mechanism against a buffered mesh design with buffer bypassing [63, 45]. We configure the buffered mesh to have 4 virtual channels (VCs) per port with 8 buffers per VC. We also compare our mechanism against CHIPPER [18], a low-complexity bufferless mesh network. We use 128-bit links for both designs. Additionally, we compare our mechanism against a flattened butterfly [32] with 4 VCs per output port, 8 buffers per VC, and 64-bit links. Our main conclusions are as follows:

\begin{tabular}{|l||l|l||l|l|}
\hline \multicolumn{1}{|l||}{ Topologies } & \multicolumn{2}{c||}{$4 \mathrm{x} 4$} & \multicolumn{2}{c|}{$8 \mathrm{x} 8$} \\
\hline & Norm. WS & Power (mWatts) & Norm. WS & Power (mWatts) \\
\hline \hline Single Ring & 0.904 & 7.696 & 0.782 & 13.603 \\
\hline Buffered HRing & 1 & 12.433 & 1 & 16.188 \\
\hline Buffered Mesh & 1.025 & 11.947 & 1.091 & 13.454 \\
\hline CHIPPER & 0.986 & 4.631 & 1.013 & 7.275 \\
\hline Flattened Butterfly & 1.037 & 10.760 & 1.211 & 30.434 \\
\hline HiRD & 1.020 & 4.746 & 1.066 & 12.480 \\
\hline
\end{tabular}

Table 6: Evaluation for $4 \times 4$ and $8 \times 8$ networks against different network designs.

1. Against designs using the mesh topology, we observe that HiRD performs very closely to the buffered mesh design both for $4 \times 4$ and $8 \times 8$ network sizes, while a buffered hierarchical ring design performs slightly worse compared to HiRD and buffered mesh designs. Additionally, HiRD performs better than CHIPPER in both $4 \times 4$ and $8 \times 8$ networks, though CHIPPER consumes less power in an $8 \times 8$ design as there is no buffer in CHIPPER.

2. Compared to a flattened butterfly design, we observe that HiRD performs competitively with a flattened butterfly in a $4 \times 4$ network, but consumes lower router power. In an 8x8 network, HiRD does not scale as well as a flattened butterfly network and performs $11 \%$ worse than a flattened butterfly network; however, HiRD consumes 59\% less power than the flattened butterfly design.

3. Overall, we conclude that HiRD is competitive in performance with the highest performing designs while having much lower power consumption. 


\section{Related Work}

To our knowledge, HiRD is the first hierarchical ring design that uses simple, deflection-based ring transfers to eliminate the need for buffering within rings while guaranteeing end-to-end packet delivery.

Hierarchical Interconnects: Hierarchical ring-based interconnect was proposed in a previous line of work [53, 64, 24, 54, 22, 29]. We have already extensively compared to past hierarchical ring proposals qualitatively and quantitatively. The major difference between our proposal and this previous work is that we propose deflection-based bridge routers with minimal buffering, and node routers with no buffering. In contrast, all of these previous works use routers with in-ring buffering, wormhole switching and flow control. Concurrent works by Kim et al. propose tNoCs, hybrid packet-flit credit-based flow control [29] and Clumsy Flow Control (CFC) [30]. However, these two designs add additional complexity because $\mathrm{tNoCs}$ [29] requires an additional credit network to guarantee forward progress while CFC requires coordination between cores and memory controllers. In contrast, flow control in HiRD is lightweight (with deflection based flow control, the Retransmit-Once mechanism, and simpler local-to-global and globalto-local buffers). Additionally, throttling decisions in HiRD can be made locally in each local ring as opposed to global decisions in CFC [30] and tNoCs [29].

Udipi et al. proposed a hierarchical topology using global and local buses [61]. While this work recognizes the benefits of hierarchy, our design builds upon a ringbased design instead of a bus-based design because a ring-based design provides better scalability. Das et al. [13] examined several hierarchical designs, including a concentrated mesh (one mesh router shared by several nearby nodes).

A previous system, SCI (Scalable Coherent Interface) [23], also uses rings, and can be configured in many topologies (including hierarchical rings). However, to handle buffer-full conditions, SCI NACKs and subsequently retransmits packets, whereas HiRD deflects only single flits (within a ring), and does not require the sender to retransmit its flits. SCI was designed for off-chip interconnect, where tradeoffs in power and performance are very different than in on-chip interconnects. The KSR (Kendall Square Research) machine [15] uses a hierarchical ring design that resembles HiRD, 
yet these techniques are not disclosed in detail and, to our knowledge, have not been publicly evaluated in terms of energy efficiency.

Other Ring-based Topologies: Spidergon [9] proposes a bidirectional ring augmented with links that directly connect nodes opposite each other on the ring. These additional links reduce the average hop distance for traffic. However, the cross-ring links become very long as the ring grows, preventing scaling past a certain point, whereas our design has no such scaling bottleneck. Octagon [28] forms a network by joining Spidergon units of 8 nodes each. Units are joined by sharing a "bridge node" in common. Such a design scales linearly. However, it it does not make use of hierarchy, while our design makes use of global rings to join local rings.

Other Low Cost Router Designs: Kim [31] proposes a low-cost router design. However, this design is explicitly designed for meshes, hence would not be directly usable in our ring-based design because of potential livelock as we discussed in [3]. Additionally, this design does not use deflections when there is contention. Mullins et al. [47] propose a buffered mesh router with single-cycle arbitration. Our work differs in that our focus is on hierarchical rings rather than meshes. Abad et al. [1] propose the Rotary Router. Their design fundamentally differs from ours because each router has an internal ring, and the network as a whole is a mesh. In contrast, HiRD's routers are simpler as they are designed for hierarchical rings. Kodi et al. [36] propose an orthogonal mechanism that reduces buffering by using links as buffer space when necessary.

Bufferless Mesh-based Interconnects: While we focus on ring-based interconnects to achieve simpler router design and lower power, other work modifies conventional buffered mesh routers by removing buffers and using deflection [5, 21, 25, 37, 46, 18, 8 , 19, 49, 50]. Applying bufferless routing principles to rings leads to inherently simpler designs, as there is only one option for deflection in a ring (i.e., continue circulating around the ring). Other works propose dropping packets under contention [21, 20]. SCARAB [25] adds a dedicated circuit-switch network to send retransmit requests. Several machines such as HEP [56], Tera [2] and the Connection Machine [26] also use deflection routing to connect different chips. 


\section{Conclusion}

We introduced $H i R D$, a simple hierarchical ring-based NoC design. Past work has shown that a hierarchical ring design yields good performance and scalability relative to both a single ring and a mesh. HiRD has two new contributions: (1) a simple router design that enables ring transfers without in-ring buffering or flow control, instead using limited deflections (retries) when a flit cannot transfer to a new ring, and (2) two guarantee mechanisms that ensure deterministically-guaranteed forward progress despite deflections. Our evaluations show that HiRD enables a simpler and lowercost implementation of a hierarchical ring network. Although an exhaustive topology comparison is not the goal of this work, our evaluations also show that HiRD is more energy-efficient than several other topologies while providing competitive performance. We conclude that HiRD represents a compelling interconnect design point to bring additional scalability to existing ring-based designs at high energy efficiency.

\section{Acknowledgments}

We thank the reviewers and SAFARI members for their feedback. We acknowledge the support of AMD, IBM, Intel, and Qualcomm. This research was partially supported by Intel Science and Technology Center on Cloud Computing, NSF (CCF 0953246 and CCF 1212962), and SRC. Rachata Ausavarungnirun is supported by the Royal Thai Government scholarship. This article is an extended and significantly revised version of a paper that appeared at SBAC-PAD 2014 [3]. Our technical report [4] includes results from both this article and our previous SBAC-PAD paper [3].

\section{References}

[1] P. Abad et al., "Rotary router: an efficient architecture for CMP interconnection networks," ISCA, 2007.

[2] R. Alverson et al., "The Tera computer system," ICS, 1990.

[3] R. Ausavarungnirun et al., "Design and evaluation of hierarchical rings with deflection routing," in SBAC-PAD, 2014.

[4] "Improving energy efficiency of hierarchical rings via deflection routing," SAFARI Technical Report TR-2014002: http://safari.ece.cmu.edu/tr.html Apr 2014.

[5] P. Baran, "On distributed communications networks," IEEE Trans. on Comm., 1964.

[6] E. Baydal et al., "A family of mechanisms for congestion control in wormhole networks," IEEE Trans. on Par. and Dist. Sys., vol. 16, 2005.

[7] C. Carrión et al., "A flow control mechanism to avoid message deadlock in k-ary n-cube networks," HiPC, 1997. 
[8] K. K.-W. Chang et al., "HAT: Heterogeneous adaptive throttling for on-chip networks," SBAC-PAD, 2012.

[9] M. Coppola et al., "Spidergon: a novel on-chip communication network," SOCC, Nov 2004.

[10] C. Craik and O. Mutlu, "Investigating the viability of bufferless NoCs in modern chip multi-processor systems," SAFARI Technical Report TR-2011-004: http://safari.ece.cmu.edu/tr.html Aug 2011.

[11] W. Dally and B. Towles, Principles and Practices of Interconnection Networks. Morgan Kaufmann, 2004.

[12] W. J. Dally and B. Towles, “Route packets, not wires: On-chip interconnection networks,” DAC, 2001.

[13] R. Das et al., "Design and evaluation of hierarchical on-chip network topologies for next generation CMPs," HPCA, 2009.

[14] R. Das, O. Mutlu, T. Moscibroda, and C. Das, "Application-aware prioritization mechanisms for on-chip networks," MICRO, 2009.

[15] T. H. Dunigan, "Kendall square multiprocessor: Early experiences and performance," in of the Intel Paragon, ORNL/TM-12194, 1994.

[16] E. Ebrahimi, C. Lee, O. Mutlu, and Y. Patt, "Fairness via source throttling: a configurable and high-performance fairness substrate for multi-core memory systems," ASPLOS, 2010.

[17] S. Eyerman and L. Eeckhout, "System-level performance metrics for multiprogram workloads," IEEE Micro, 2008.

[18] C. Fallin et al., "CHIPPER: A low-complexity bufferless deflection router," HPCA, 2011.

[19] —_ "MinBD: Minimally-buffered deflection routing for energy-efficient interconnect," NOCS, 2012.

[20] C. Gómez et al., "BPS: a bufferless switching technique for NoCs," Wina, 2008.

[21] _ _ "Reducing packet dropping in a bufferless NoC," EuroPar, 2008.

[22] R. Grindley et al., "The NUMAchine multiprocessor,” ICPP, 2000.

[23] D. Gustavson, "The scalable coherent interface and related standards projects," IEEE Micro, 1992.

[24] V. C. Hamacher and H. Jiang, "Hierarchical ring network configuration and performance modeling," IEEE Transaction on Computers, 2001.

[25] M. Hayenga et al., "Scarab: A single cycle adaptive routing and bufferless network," MICRO, 2009.

[26] W. Hillis, The Connection Machine. MIT Press, 1989.

[27] Intel Corporation, "Intel details 2011 processor features," http://newsroom.intel.com/community/intel_newsroom/blog/2010/09/13/intel-details-2011-processor-features-offers-stunning-visuals-built-in

28] F. Karim et al., "On-chip communication architecture for OC-768 network processors," DAC, 2001.

[29] H. Kim et al., "Transportation-network-inspired network-on-chip," HPCA, 2014.

[30] —_ "Clumsy flow control for high-throughput bufferless on-chip networks," IEEE CAL, 2013.

[31] J. Kim, "Low-cost router microarchitecture for on-chip networks," MICRO, 2009.

[32] J. Kim and W. Dally, "Flattened butterfly: A cost-efficient topology for high-radix networks," ISCA, 2007.

[33] J. Kim and H. Kim, "Router microarchitecture and scalability of ring topology in on-chip networks," NoCArc, 2009.

[34] Y. Kim, D. Han, O. Mutlu, and M. Harchol-Balter, “ATLAS: a scalable and high-performance scheduling algorithm for multiple memory controllers," HPCA, 2010.

[35] Y. Kim, M. Papamichael, O. Mutlu, and M. Harchol-Balter, "Thread cluster memory scheduling," MICRO, 2010.

[36] A. Kodi et al., "iDEAL: Inter-router dual-function energy and area-efficient links for network-on-chip (NoC) architectures," ISCA, 2008.

[37] S. Konstantinidou and L. Snyder, "Chaos router: architecture and performance," ISCA, 1991.

[38] D. Kroft, “Lockup-free instruction fetch/prefetch cache organization,” ISCA, 1981.

[39] H. Kwak, C. Lee, H. Park, and S. Moon, "What is Twitter, a social network or a news media?" 2010.

[40] A. Kyrola, G. Blelloch, and C. Guestrin, "GraphChi: Large-scale graph computation on just a PC,” 2012. 
[41] J. Laudon and D. Lenoski, "The SGI Origin: a ccNUMA highly scalable server," ISCA, 1997.

[42] H. Lee et al., "Cloudcache: Expanding and shrinking private caches," HPCA, 2011.

[43] Y. Low, J. Gonzalez, A. Kyrola, D. Bickson, C. Guestrin, and J. M. Hellerstein, "GraphLab: A new parallel framework for machine learning," 2010.

[44] C.-K. Luk et al., "Pin: building customized program analysis tools with dynamic instrumentation," PLDI, 2005.

[45] G. Michelogiannakis et al., "Evaluating bufferless flow-control for on-chip networks," NOCS, 2010.

[46] T. Moscibroda and O. Mutlu, "A case for bufferless routing in on-chip networks," ISCA, 2009.

[47] R. Mullins et al., "Low-latency virtual-channel routers for on-chip networks," ISCA, 2004.

[48] S. P. Muralidhara, L. Subramanian, O. Mutlu, M. Kandemir, and T. Moscibroda, "Reducing memory interference in multicore systems via application-aware memory channel partitioning," MICRO, 2011.

[49] G. P. Nychis et al., "Next generation on-chip networks: What kind of congestion control do we need?" Hotnets, 2010.

[50] - "On-chip networks from a networking perspective: Congestion and scalability in many-core interconnects," SIGCOMM, 2012

[51] D. Pham et al., "Overview of the architecture, circuit design, and physical implementation of a first-generation CELL processor," JSSC, 2006.

[52] Rachata Ausavarungnirun and others, "NOCulator," https://github.com/CMU-SAFARI/NOCulator

[53] G. Ravindran and M. Stumm, "A performance comparison of hierarchical ring- and mesh-connected multiprocessor networks," HPCA, 1997.

[54] - , "On topology and bisection bandwidth for hierarchical-ring networks for shared memory multiprocessors," HPCA, 1998.

[55] L. Seiler et al., "Larrabee: a many-core x86 architecture for visual computing," SIGGRAPH, 2008.

[56] B. Smith, "Architecture and applications of the HEP multiprocessor computer system," SPIE, 1981.

[57] A. Snavely and D. M. Tullsen, "Symbiotic jobscheduling for a simultaneous multithreaded processor," ASPLOS, 2000.

[58] C. Sun et al., "DSENT - a tool connecting emerging photonics with electronics for opto-electronic networks-on-chip modeling," NOCS, 2012.

[59] M. Thottethodi, A. Lebeck, and S. Mukherjee, "Self-tuned congestion control for multiprocessor networks," HPCA, 2001.

[60] S. Tota et al., "Implementation analysis of NoC: a MPSoC trace-driven approach," GLSVLSI, 2006.

[61] A. N. Udipi et al., "Towards scalable, energy-efficient, bus-based on-chip networks," HPCA, 2010.

[62] H. Vandierendonck and A. Seznec, "Fairness metrics for multi-threaded processors," IEEE Computer Architecture Letters, Feb 2011.

[63] H. Wang et al., "Power-driven design of router microarchitectures in on-chip networks," MICRO, 2003.

[64] X. Zhang and Y. Yan, "Comparative modeling and evaluation of CC-NUMA and COMA on hierarchical ring architectures," IEEE TPDS, 1995. 